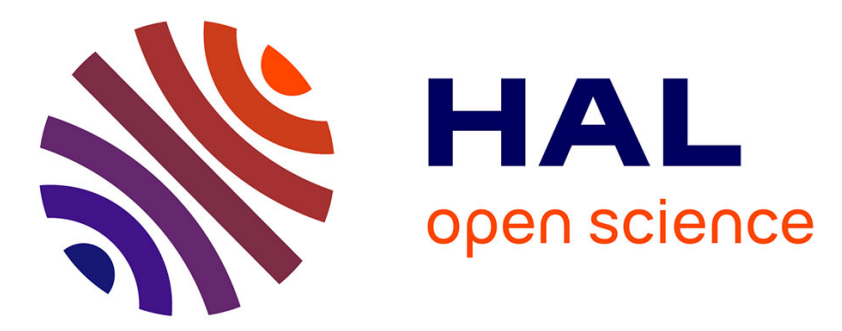

\title{
Etude in vitro des facteurs responsables de la formation des populations femelles de pathotypes français d'Heterodera avenae Woll. sur variétés d'orge et de blé
} Françoise Person-Dedryver, Danielle Pannetier

\section{- To cite this version:}

Françoise Person-Dedryver, Danielle Pannetier. Etude in vitro des facteurs responsables de la formation des populations femelles de pathotypes français d'Heterodera avenae Woll. sur variétés d'orge et de blé. Agronomie, 1987, 7 (10), pp.779-788. hal-00884953

\section{HAL Id: hal-00884953 https://hal.science/hal-00884953}

Submitted on 1 Jan 1987

HAL is a multi-disciplinary open access archive for the deposit and dissemination of scientific research documents, whether they are published or not. The documents may come from teaching and research institutions in France or abroad, or from public or private research centers.
L'archive ouverte pluridisciplinaire HAL, est destinée au dépôt et à la diffusion de documents scientifiques de niveau recherche, publiés ou non, émanant des établissements d'enseignement et de recherche français ou étrangers, des laboratoires publics ou privés. 


\section{Etude in vitro des facteurs responsables de la formation des populations femelles de patho- types français d'Heterodera avenae Woll. sur variétés d'orge et de blé}

Françoise PERSON-DEDRYVER

avec la collaboration technique de Danielle PANNETIER

I.N.R.A., Laboratoire de Zoologie, Centre de Recherches de Rennes, B.P. 29, F 35650 Le Rheu

La formation des femelles est étudiée sur plante poussant en conditions entièrement contrôlées de boîtes de Petri, dans le but de minimiser l'impact des facteurs méthodologiques sur le développement du nématode. Le milieu étant constitué uniquement d'eau et d'agar agar, les conditions de culture sont peu favorables au développement des plantes. Nous avons donc été amenés à étudier le facteur poids du grain sur la formation des femelles, le grain constituant la seule réserve nutritive possible pour la plante. Il n'intervient en fait que pour une seule variété sur les 11 lignées de blé étudiées de ce point de vue. Les conditions de culture de la plante productrice de grains à tester sembleraient également avoir une influence sur le développement des femelles. Un géniteur non fixé comme hôte à l'encontre d'H. avenae, tel que la lignée de blé V.P.M. 1.1.1.2. R4 peut être responsable de variations de l'importance des populations femelles formées.

La comparaison des résultats obtenus sur divers haplö̈des doublés issus d'un même blé ou entre haploïdes doublés et leur lignée d'origine montre que les femelles se forment en moyenne de manière similaire sur ces diverses plantes présentant des caractères hôtes génotypiquement identiques vis-à-vis d'H. avenae.

Par contre, l'emploi de grains issus d'un même haploïde doublé de blé, matériel végétal génotypiquement homogène, ne supprime pas les variations dans la formation des femelles entre les répétitions d'un même essai. Ces variations dépendent donc, soit de la méthodologie employée, soit du nématode lui-même.

La plus grande part de la variabilité enregistrée dans la formation des femelles dépend du nématode lui-même. Chaque pathotype d' $H$. avenae possède une agressivité propre à l'encontre de chaque lignée testée. Cette agressivité peut être modifiée, soit par recombinaisons génétiques après croisement entre pathotypes, soit par pression de sélection sur plante possédant des gènes de résistance vis-à-vis du nématode. A partir de nos résultats, nous pouvons avancer l'hypothèse suivante : l'utilisation effective de la capacité d'un pathotype à contourner des résistances se traduit par une diminution globale de son agressivité.

Mots clés additionnels : Nématode, Triticum aestivum, Hordeum vulgare L., haploïde doublé, agressivité, hybridation, pression de sélection. pathotypes of Heterodera avenae Woll. on wheat and barley varieties.

The development of females has been studied on young cereal seedlings grown in Petri dishes. In these controlled conditions of cereal culture, the impact of physical factors on nematode development was minimal. The culture medium consisted of water and agar, so the seeds were the main nutritive reserve of the plants. The weight factor of the seed had an influence on the development of females for only one variety of the 11 tested wheat lines. The culture conditions of the plants from which the seed was taken were sometimes responsible for variable formation of female populations.

The temperature fluctuations registered between Petri dishes stocked in the culture room did not induce variable development of the females. In the case of a wheat parent variable for host quality to $H$. avenae, such as V.P.M. 1.1.1.2. R4, fluctuation of female populations was also observed.

The results obtained on genotypically identical seeds of different doubled haploids issued from the same wheat cultivar or on their original line showed that the mean numbers of females developed on these different plants were statistically similar. However, the use of genotypically identical seeds issued from the same doubled haploid of wheat did not remove all variability in female formation in the replicates of a single trial. The nematode itself provides the main source of variability in female development. Each pathotype of $H$. avenae had its own aggressiveness against each cereal line tested. Modifications of this aggressiveness were obtained by genetic recombination in crosses between different pathotypes or by selection pressure on plants resistant to the nematode. From our results, it appears that the aggressiveness of a pathotype tends to decrease as a consequence of more effective virulence in relation to resistance genes.

Additional key words : Nematode, Triticum aestivum, Hordeum vulgare L., doubled haploid, aggressiveness, hybridization, selection pressure. 


\section{INTRODUCTION}

Le caractère fluctuant de l'importance des populations femelles d' $H$. avenae est noté lors de la réalisation de tout test de multiplication du nématode fait, soit à l'extérieur sur sol contaminé, soit en conditions contrôlées de boîtes de Petri sur agar agar (RIVOAL, 1977 ; Person \& Doussinault, 1978). Certains facteurs responsables de cette fluctuation enregistrée dans la formation des femelles sont déjà bien connus. Le plus important concerne l'existence de différents pathotypes du nématode répertoriés en France (RIVOAL, 1977) se multipliant plus ou moins sur plantes dépourvues de gènes de résistance vis-à-vis d' $H$. avenae (RIVOAL, 1977 ; PERSON \& DOUSSINAULT, 1978 ; RIVOAL \& Person-Dedryver, 1982). Les variations de température enregistrées durant le développement des différents pathotypes ont également un effet non négligeable sur l'importance des populations femelles formées (PERSON \& DoussinaulT, 1978).

Une étude explicative des phénomènes jouant sur les variations enregistrées entre divers niveaux de populations femelles d' $H$. avenae obtenus sur une même variété a été entreprise. Nous voulons ainsi mettre en évidence l'influence de facteurs essentiellement biologiques ou parfois physiques responsables de la fluctuation de développement d' $H$. avenae enregistrée sur cultivar d'orge ou de blé. L'origine et la nature du matériel végétal et du nématode ont été analysées successivement. Pour la réalisation de ce travail, la méthode d'élevage du nématode en conditions contrôlées des boîtes de Petri a été préférée à celles utilisant du sol naturellement ou artificiellement contaminé par des kystes. Cette méthode permet l'étude du développement d'un nombre déterminé de larves infectieuses L2 jusqu'au stade femelle adulte fécond, et ce type d'analyse ne peut être réalisé avec les techniques se basant sur l'inoculation par kyste avec taux de remplissage et de sortie de larves fluctuants et invérifiables (RIVOAL et al., 1978).

Dans le but de minimiser l'intervention des facteurs inhérents à la méthode utilisée sur les résultats obtenus entre chaque répétition ou essai, un travail préliminaire concernant essentiellement les variations de la température et le poids des grains a été entrepris. 11 complète les études menées dans ce sens par PERSON \& DOUSSINAULT (1978).

\section{MATÉRIEL ET MÉTHODES}

\section{A. Le nématode}

Trois pathotypes d'origine différente seront utilisés : FR1 méridional, FR4 septentrional, FR2 de CharentePoitou et identifiés pour les 2 premiers aux pathotypes Ha41 et Hal2 déterminés sur la gamme d'hôtes internationale d' $H$. avenae et répertoriés par ANDERSEN \& ANDERSEN (1982).

Les kystes utilisés pour cette étude proviennent, quand aucune précision n'est apportée, de culture de blé cv. Hardi réalisée en pot sur sol infesté en conditions extérieures. Ils sont conservés dans de l'eau durant 2 mois à $20^{\circ} \mathrm{C}$, puis à $5{ }^{\circ} \mathrm{C}$ afin de lever la diapause des larves infectieuses L2 (RIVOAL, 1979).
Dans le but d'élargir ou de mettre en évidence les potentialités de variation de l'agressivité du parasite, des croisements entre pathotypes et des pressions de sélection ont été réalisés. La pression de sélection est faite au moyen d'élevages de nématodes issus d'un pathotype donné sur au moins 2 cultures consécutives de la même variété présentant ou non des gènes de résistance vis-à-vis d'H. avenae. Les hybrides $\mathrm{F} 1$ issus de 2 pathotypes sont obtenus suivant la technique décrite précédemment (PERSON \& RIVOAL, 1979).

\section{B. Le végétal}

Quatre cultivars de blé : Triticum aestivum, cvs "Capitole ", " Hardi ", "Lutin », " Moisson " et un d'orge : Hordeum vulgare L. cv. "Aramir » sont utilisés. Pour ces 5 variétés démunies de gènes de résistance connus à l'égard d' $H$. avenae, deux composantes du test pourraient être à l'origine de variations éventuelles de l'importance des populations femelles dans nos travaux : le poids du grain et la plante d'où proviennent les grains. Le grain est considéré, dans cette expérimentation comme un apport nutritif essentiel à la jeune plantule poussant sur milieu constitué uniquement d'eau et d'agar. Les plantes, dont sont issus les grains étudiés, ont été cultivées en serre afin d'avoir des conditions de culture les plus homogènes possibles. Plusieurs épis par variété sont étudiés, chacun d'eux provient de plantes différentes.

Pour étudier l'effet de l'homogénéité des génotypes pour leur caractère hôte vis-à-vis d' $H$. avenae, nous avons comparé différentes lignées d'haploïdes doublés des variétés de blé cvs "Athys », "César » et " Moisson », soit entre elles, soit à leur lignée d'origine. Les haplö̈des doublés sont produits et fournis par E. PICARD de l'université de Paris Sud centre d'Orsay, et sont obtenus par cultures d'anthères de ces cultivars. Leur nomenclature sera la suivante :

$\begin{array}{cccc}\text { HD } & (\mathrm{x}) & \mathrm{Y} & \mathrm{gn} \\ \begin{array}{c}\text { haploïde } \\ \text { doublé }\end{array} & \begin{array}{c}\text { génotype } \\ \text { haploïdisé }\end{array} & \begin{array}{c}\mathrm{n}^{\circ} \text { de } \\ \text { l'haplö̈de } \\ \text { doublé de ce } \\ \text { génotype }\end{array} & \begin{array}{c}\text { génération } \\ \text { d'autofécon- } \\ \text { dation après } \\ \text { haplö̈disation }\end{array}\end{array}$

Ces haploïdes doublés sont certainement fixés pour la plupart des gènes. Cependant différents haplö̈des doublés issus de « Moisson » ont présenté des réponses quantitatives variées à l'infection par Erysiphe graminis D. C., Fusarium roseum Link. et Cercosporella herpotrichoides Fron. (PARISI \& PICARD, 1986). Ces variations de la réponse à l'égard d'agents pathogènes devraient trouver une explication dans les changements moléculaires notés chez le DNA ribosomal de ces haploïdes doublés (RODE et al., 1987).

Pour voir dans quelle mesure il est possible de sélectionner dans un géniteur qui présente une hétérogénéité de sa capacité à multiplier $H$. avenae, nous avons introduit dans l'expérience la lignée de blé V.P.M. 1.1.1.2. R4 issue du croisement (Aegilops ventricosa 11 $\times$ Triticum persicum) $\times$ Marne $^{3}$ (DOSBA \& DOUSSINAULT, 1978). Cette lignée pourrait hériter de la résistance de l'Aegilops ventricosa Tausch $\mathrm{N}^{\circ} 11$ (DosBA \& RIVOAL, 1981) et présente une grande variabilité de son caractère hôte vis-à-vis d' $H$. avenae (DOSBA et al., 1979). 
Le cultivar d'orge cv. "Ortolan » présentant des gènes de résistance efficaces à l'encontre de 2 pathotypes d' $H$. avenae $\mathrm{Ha} 41$ et $\mathrm{Fr} 3$ (Ha11) (PERSONDEDRYVER \& DOUSSINAULT, 1984) sera utilisé dans le cadre d'étude de pression de sélection.

\section{Les tests nématologiques}

Les racines des plantes cultivées en boîtes de Petri sur milieu gélosé ( $20 \mathrm{~g}$ agar/1 d'eau) sont infectées par les larves infectieuses L2 du nématode suivant la technique PERSON \& Doussinault (1978). Pour révéler le caractère multiplicateur des plantes hôtes, nous avons démontré qu'il existe un effet inoculum. Il est nécessaire d'apporter 48 larves du nématode pour le pathotype Ha 12 et seulement 24 pour les 2 autres Ha41 et Fr2.

Après inoculation, les boîtes sont stockées $24 \mathrm{~h}$ à $20{ }^{\circ} \mathrm{C}$ pour favoriser la pénétration des larves et dans ces conditions environ 80 p. 100 d'entre elles sont assurées d'entrer dans les racines de chaque plante de l'essai. Les boîtes sont ensuite placées pour 2 mois dans des enceintes climatisées à grande capacité de stockage avec une photopériode de $16 \mathrm{~h}$ d'éclairage. La température d'expérimentation est alors choisie en fonction de l'optimum de développement de chaque pathotype et varie en fonction des périodes d'obscurité et d'éclairage, entre 14 et $19{ }^{\circ} \mathrm{C}$ pour $\mathrm{Ha} 41$ et 14 et $16{ }^{\circ} \mathrm{C}$ pour $\mathrm{Ha} 12$ et $\mathrm{Fr} 2$. Chaque essai est stocké indépendemment.

Pour réduire la place occupée, on a eu recours à des empilements de 4 boîtes dont la position respective est repérée par une lettre de $\mathrm{A}$ à $\mathrm{D}$ en allant de haut en bas (fig. 1). Une cinquième boîte cultivée est déposée sur la

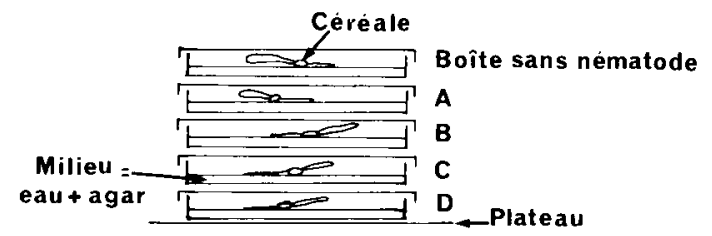

Figure 1

Position de la boîte de Petri dans la pile : $A, B, C$ ou $D$. Place of the Petri dish in the pile: $A, B, C$ ou $D$.

pile, mais aucun nématode n'y sera inoculé. Après réalisation de diverses manipulations, il s'avère que cette façon d'opérer supprime toute condensation sur le couvercle de la boîte en position $\mathrm{A}$ et évite ainsi une déshydratation excessive de la gélose de cette boîte, néfaste à l'expérimentation. Ce facteur position de la boîte, non étudié antérieurement (PERSON \& DOUSSINAULT, 1978), pourrait jouer un rôle non négligeable dans la formation des femelles du parasite par l'intermédiaire de la variation de la température et (ou) de la réaction des plantes à la quantité de lumière reçue entre les différentes positions A, B, C et D.

\section{Enregistrement de la température dans les boîtes de Petri}

L'empilement des boîtes par pile de 4 , a nécessité l'enregistrement de la température dans une série de
4 boîtes pour évaluer les fluctuations des amplitudes thermiques suivant les positions A, B, C et D des boîtes (fig. 1). Nous avons choisi de faire l'enregistrement, dans l'expérimentation correspondant à l'étude du pathotype Ha41 (14 à $19^{\circ} \mathrm{C}$ ) qui présente au thermomètre un maximum d'amplitude thermique entre les périodes éclairées ou non.

Les mesures de la température dans les 4 boîtes sont effectuées par P. BAUTRAIS (Laboratoire I.N.R.A. Zoologie Rennes) à l'aide de thermocouples reliés à une centrale de mesure enregistrant les résultats toutes les 2 heures.

\section{E. Analyses statistiques}

Les études de l'influence des facteurs position de la boîte dans la pile, origines des plantes testées et du nématode utilisé, sur la formation des femelles d' $H$. avenae sont basées sur l'analyse de variance avec ou sans facteur hiérarchique. L'utilisation de l'extension du test à rang multiple pour moyennes à nombres de répétitions inégaux (KRAMER, 1956) ou le test de Duncan permettent la classification des moyennes de femelles adultes fécondes formées, quand cela s'avère nécessaire.

La formation des femelles en fonction du poids du grain est basée sur une analyse de régression et corrélation linéaire.

\section{LES RÉSULTATS}

Le nombre de femelles formées peut dépendre soit de la méthode employée, soit de l'origine du végétal ou de celle du nématode. Les trois facteurs seront analysés successivement.

\section{A. Influence de la méthode utilisée}

Les plantes sont cultivées dans les conditions particulières de la boîte qui leur sont défavorables. De ce fait les facteurs position de la boîte, poids du grain et origine de l'épi utilisé, jamais étudiés jusqu'à présent, peuvent jouer sur les résultats et sont pris en compte dans l'estimation de l'influence des facteurs méthodologiques sur la formation des femelles.

\section{Influence de la position de la boîte de Petri}

\section{a) Etude de l'enregistrement de la température}

Dans les différentes boîtes (fig. 2) deux phases « plateaux " sont obtenues au cours de $24 \mathrm{~h}$ de culture et correspondent aux périodes obscures et éclairées. $\mathrm{Si}$ elles sont pratiquement identiques et comprises entre $14,1{ }^{\circ} \mathrm{C}$ et $14,4^{\circ} \mathrm{C}$ pour les 4 positions de boîte de Petri en période non éclairée, des différences importantes sont enregistrées avec éclairage. La boîte en position A passe par la température maximum $\left(19,1^{\circ} \mathrm{C}\right)$. L'effet de l'éclairage se fait de moins en moins sentir, lorsque sont considérées successivement les positions $B, C$ puis $D$. Les positions $B$ et $C$ présentent des maxima thermiques voisins et proches de 


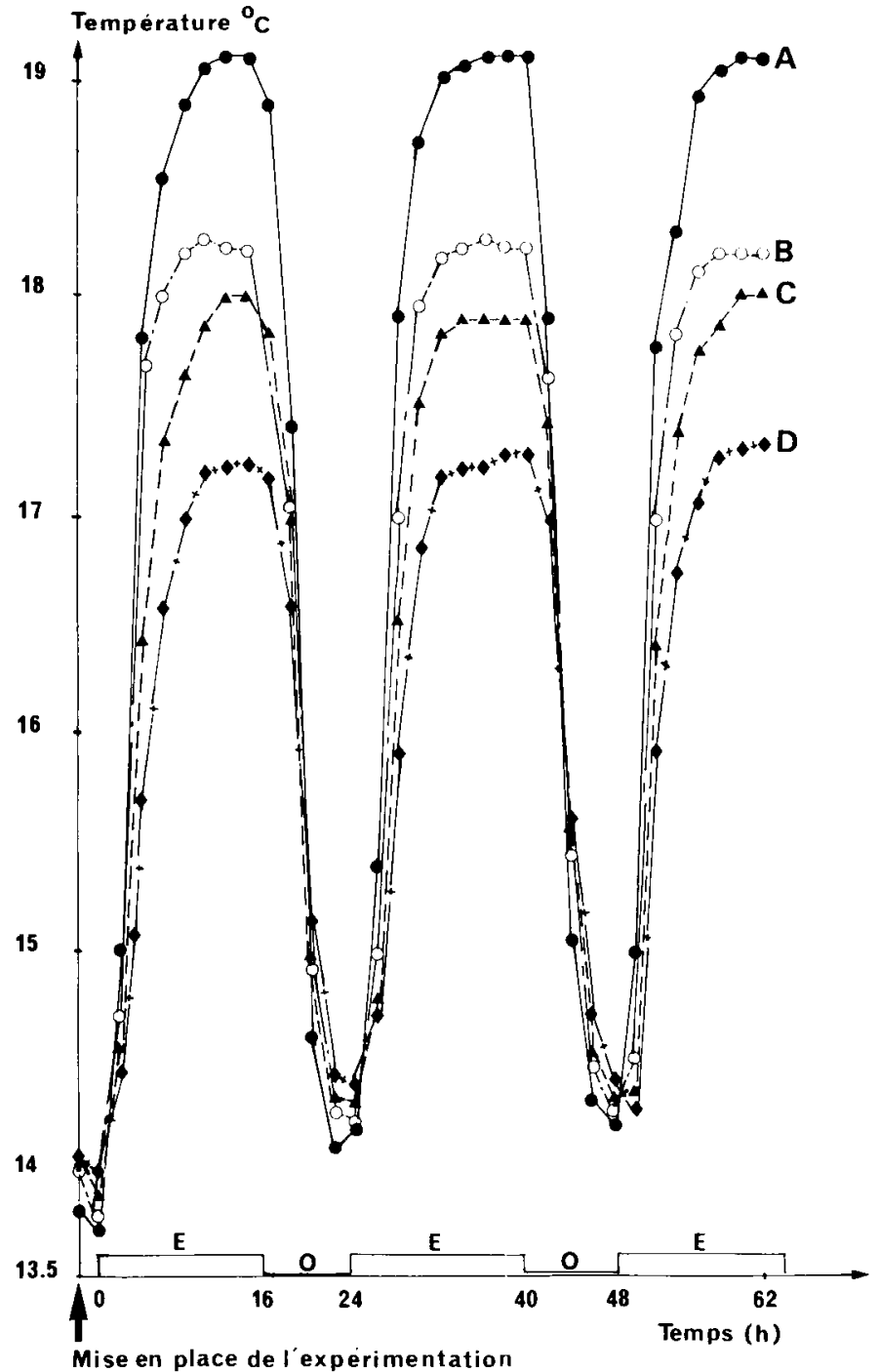

$18^{\circ} \mathrm{C}$, alors que pour la position $\mathrm{D}$, la température atteint difficilement $17,3{ }^{\circ} \mathrm{C}$. Il y a donc pratiquement $2{ }^{\circ} \mathrm{C}$ de différence pendant la période de lumière entre les positions A et $\mathrm{D}$. Ces 2 types de boîte passent par des températures minimales très voisines. Ainsi nous estimons à presque $1{ }^{\circ} \mathrm{C}$ jour la différence dans les sommes de température qu'elles reçoivent journellement.

Les amplitudes restant identiques 2 jours consécutifs, nous avons arrêté l'enregistrement $62 \mathrm{~h}$ après sa mise en place.

\section{b) Etude de la formation des femelles (tabl. 1)}

Pour un pathotype et une variété donnée, le nombre de femelles formées peut être très différent d'une plante à une autre : de 1 à 13 pour Hardi en ce qui concerne Ha4l par exemple. Mais en aucun cas, nous ne pouvons déceler une différence significative entre les moyennes de femelles développées sur les 4 catégories de boîtes : A, B, C ou D pour un cultivar donné et un pathotype déterminé. Ces moyennes fluctuent cependant légèrement entre elles. La position $\mathrm{C}$ donne cependant la moyenne de femelles $\mathrm{Ha} 41$ la plus élevée sur les 3 cultivars testés vis-à-vis de ce pathotype.

\section{Figure 2}

Enregistrement de la température dans 4 boîtes de Petri de niveaux différents: $A, B, C$ ou $D$.

$E=$ éclairage $O=$ obscurité.

Temperature registered in 4 Petri dishes from different places in the pile : $A, B, C$ or $D$.

$E=$ light $; O=$ derk

TABLEAU 1

Etude du nombre de femelles d'Heterodera avenae formées par plante en fonction de la position de la boîte dans la pile. Production of females of Heterodera avenae per plant according to the place of the Petri dish in the pile.

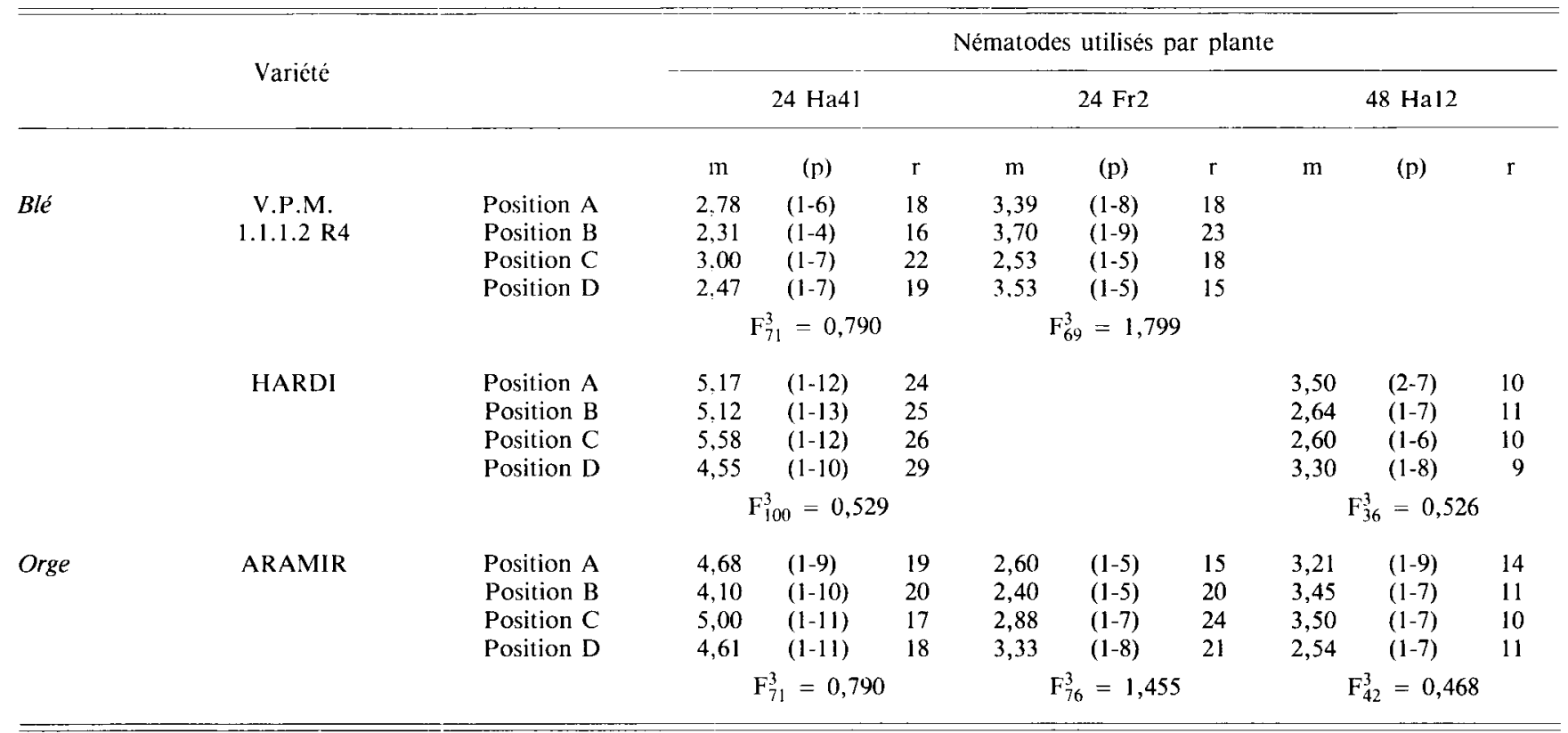

$\mathrm{m}=$ moyenne $;(\mathrm{p})=$ valeurs extrêmes minimales et maximales $; \mathrm{r}=$ nombre de répétitions $; \mathrm{F}_{\mathrm{k} 2}^{\mathrm{k}}=\mathrm{F}$ de Snedecor calculé et présentant $\mathrm{k}_{1}$, degrés de liberté du facteur étudié (ici position de la boîte) $k_{2}$ degrés de liberté de la résiduelle.

$\mathrm{m}=$ mean $;(\mathrm{p})=$ maximum and minimum values $; \mathrm{r}=$ replicates $; \mathrm{F}_{\mathrm{k} 2}^{\mathrm{k}}=\mathrm{F}$ Snedecor. 
TABLEAU 2

Formation des femelles d'Heterodera avenae en boîtes de Petri en fonction du poids du grain. Production of females of Heterodera avenae in Petri dishes according to seed weight.

\begin{tabular}{|c|c|c|c|c|c|c|c|c|c|}
\hline \multicolumn{3}{|c|}{ Variété de Blé } & \multicolumn{7}{|c|}{ Nématodes utilisés par plante } \\
\hline & & \multicolumn{4}{|c|}{$24 \mathrm{Ha} 4 \mathrm{l}$} & \multicolumn{4}{|c|}{$24 \mathrm{Fr} 2$} \\
\hline & & \multirow{2}{*}{$\begin{array}{c}\text { Nbre de } \\
\text { de grains } \\
\text { par épi }\end{array}$} & \multirow{2}{*}{$\begin{array}{l}\text { Femelles } \\
\text { formées/plante } \\
\begin{array}{ll}m & \text { (p) }\end{array}\end{array}$} & \multicolumn{2}{|c|}{ Poids du grain $(\mathrm{g})$} & \multirow{2}{*}{$\begin{array}{c}\text { Nbre de } \\
\text { de grains } \\
\text { par épi }\end{array}$} & $\begin{array}{c}\text { Femelles } \\
\text { formées/plante }\end{array}$ & \multicolumn{2}{|c|}{ Poids du grain $(\mathrm{g})$} \\
\hline & & & & $\mathrm{m}$ & (p) & & $\mathrm{m} \quad(\mathrm{p})$ & $\mathrm{m}$ & (p) \\
\hline \multirow[t]{6}{*}{ HARDI } & épi 1 & 29 & $3,76 \quad(1-8)$ & 0,029 & $(0,010-0,040)$ & 35 & $3,06 \quad(1-8)$ & $0,024^{c}$ & $(0,010-0,035)$ \\
\hline & épi 2 & 38 & $3,76 \quad(1-10)$ & 0,027 & $(0,010-0,040)$ & 44 & $2,41 \quad(1-7)$ & $0,027^{b c}$ & $(0,010-0,045)$ \\
\hline & épi 3 & 27 & $3,70 \quad(1-10)$ & 0,025 & $(0,005-0,040)$ & 42 & $2,38 \quad(1-5)$ & $0,032^{\mathrm{a}}$ & $(0,020-0,040)$ \\
\hline & épi 4 & 29 & $2,72 \quad(1-8)$ & 0,025 & $(0,010-0,045)$ & 39 & $3,15 \quad(1-8)$ & $0,028^{b}$ & $(0,010-0,040)$ \\
\hline & épi 5 & 38 & $3,24 \quad(1-7)$ & 0,024 & $(0,010-0,040)$ & & & & \\
\hline & & & $\mathrm{F}_{156}^{4}=1,424$ & \multicolumn{2}{|c|}{$F_{156}^{4}=1,894$} & & $F_{156}^{3}=2,424$ & \multicolumn{2}{|c|}{$\mathrm{F}_{156}^{3}=7,412^{* * *}$} \\
\hline
\end{tabular}

$\begin{array}{lllllll}\text { CAPITOLE } & \text { épi 1 } & 34 & 2,76^{\mathrm{b}} & (1-5) & 0,048^{\mathrm{a}} & (0,010-0,070) \\ & \text { épi 2 } & 29 & 3,10^{\mathrm{b}} & (1-7) & 0,038^{\mathrm{b}} & (0,010-0,060) \\ & \text { épi 3 } & 42 & 4,57^{\mathrm{a}} & (1-9) & 0,030^{\mathrm{c}} & (0,010-0,050) \\ & & & F_{102}^{2}=13,828^{* * *} & F_{102}^{2} & =19,60^{* * *}\end{array}$

Corrélation entre nombre de femelles/plante et poids du grain : $r=0,245^{*}$ pour 104 degrés de liberté

\footnotetext{
$\mathrm{m}=$ moyennes $;(\mathrm{p})=$ valeurs extrêmes minimale ou maximale ; $\mathrm{F}$ de SNEDECor significatif au seuil $0,001 * * *$

Test de Kramer = les valeurs affectées par la même lettre ne diffèrent pas statistiquement entre elles au seuil 0,05 .

$r=$ coefficient de corrélation significatif au seuil $0,05^{*}$.

$\mathrm{m}=$ mean $;(\mathrm{p})=$ maximum and minimum values; $\mathrm{F}$ significant at $\mathrm{P}=0.001^{* * *}$.

Values with the same letter are not significantly different at $\mathrm{P}=0.05$ according to Kramer's test.

$r=$ correlation coefficient significant at $\mathrm{P}<0.05^{*}$.
}

\section{Influence du poids du grain (tabl. 2)}

Pour le cultivar «Hardi" et le pathotype Fr2 aucune différence statistique n'est décelée entre les nombres moyens de femelles formées sur divers épis présentant des poids moyens de leurs grains différents. Les coefficients de corrélation calculés entre les nombres de femelles développées par plante et le poids du grain pour l'ensemble des grains testés par rapport à un pathotype donné Ha41 ou Fr2 ne sont jamais significatifs.

Pour "Capitole » et le pathotype Ha41, le nombre moyen de femelles par épi est inversement proportionnel au poids moyen des grains par épi. Une corrélation linéaire négative significative au seuil 0,02 lie dans ce cas le nombre de femelles à celui du poids du grain.

La fluctuation du poids du grain est plus importante chez « Capitole » $(0,010$ à $0,070 \mathrm{~g})$ que chez « Hardi » $(0,005$ à $0,050 \mathrm{~g})$.

\section{B. Influence du végétal}

1. Etude de matériel végétal génotypiquement homogène : les haploïdes doublés d'《 Athys », "César » et « Moisson ».

A l'aide du pathotype Ha41, nous avons analysé 2 facteurs inhérents au végétal et pouvant influencer la formulation des femelles : l'origine des plantes haploïdisées d'un même génotype, le niveau de générations d'autofécondation après haploïdisation (tabl. 3).
Pour un haploïde doublé donné issu de « Moisson » : HD (Moisson) $2 \mathrm{~g} 3$ le facteur origine de la plante n'influe pas sur la formation moyenne des femelles. Seules des différences sont enregistrées entre poids des grains issus de 2 épis d'une même plante.

Le blé « Moisson » et 2 haploïdes doublés différents issus de Moisson HD (Moisson) 1 g3 et HD (Moisson) $3 \mathrm{~g} 3$ sont à l'origine d'une formation moyenne de femelles similaire, bien que les poids moyens des grains soient parfois significativement différents d'un épi à un autre. Dans ces 2 essais le nombre de femelles formées n'est donc pas lié au facteur poids du grain testé.

Avec l'haploïde HD (César) 1 le facteur génération est testé. Le nombre de femelles formées et le poids moyen des grains de la $7^{\mathrm{e}}$ génération sont significativement supérieurs à ceux de la $6^{\mathrm{e}}$ génération. Aucune corrélation n'est cependant notée dans ce cas entre les nombres de femelles formées par plante et les poids des grains.

Un fait important est à souligner : l'emploi d'haploïde ne supprime pas les variations importantes du nombre de femelles formées entre chaque répétition d'un même épi et les écarts vont de 1 à 7 aussi bien pour les haplö̈des issus de "César " que pour ceux provenant de «Moisson».

Une dernière expérimentation faite avec les pathotypes Fr2 et Ha12 démontre à l'aide d'effectifs de plantes plus réduits que la formation des femelles sur des haploïdes issus d' "Athys » ou sur leur lignée mère n'est pas influencée par la nature de la plante utilisée ou le niveau de génération d'autofécondation après haploïdisation (tabl. 4). 
TABLEAU 3

Etude de la formation des femelles d'Heterodera avenae, pathotype Ha4l, sur des haploides doublés de blé. Production of females of Heterodera avenae (pathotype Ha4I) on the doubled haploid issued from wheat.

\section{Haploïde - Doublé étudié}

Nombre de grains/épis
Femelles formées/plantes
Poids du grain (g)

Haploïdes issus de MOISSON

m

(p)

m

(p)

1) Etude de plantes génotypiquement

identiques issues de :

HD (MOISSON) $2 \mathrm{~g} 3$

$\begin{array}{ll}\text { plante } n^{\circ} 1 & \text { épi } 1 \\ \text { plante } n^{\circ} 2 & \text { épi } 2 \\ & \text { épi } 1 \\ & \text { épi } 2\end{array}$

analyse de variance (hiérarchique) pi 1

1
Facteur plante $\mathrm{F}_{95}^{\mathrm{l}}=2,104$

Facteur épi $\quad F_{95}^{2}=1,992$

Corrélation entre nombre de femelles/plante et poids du grain $r=-0,109$ pour 98 degrés de liberté

2) Etude de plantes différentes issues de :

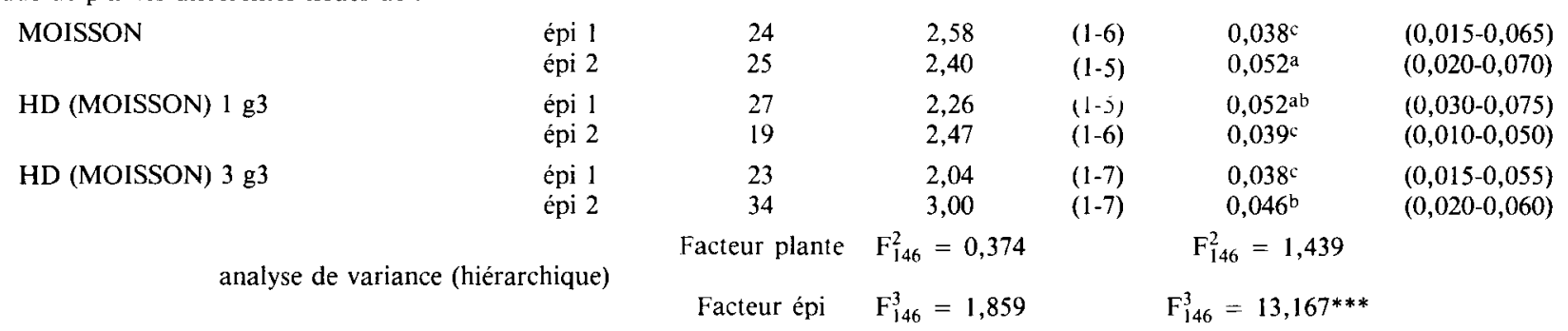

Corrélation entre nombre de femelles/plante et poids du grain $r=0,094$ pour 151 degrés de liberté

Haplö̈des issus de CÉSAR

Etude du niveau de génération

d'autofécondation après haploïdisation

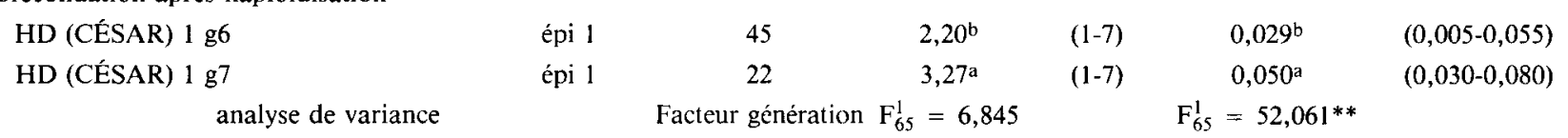

Corrélation entre nombre de femelles/plante et poids du grain $r=-0,034$ pour 66 degrés de liberté

$\mathrm{m}=$ moyenne $;(\mathrm{p})=$ valeurs extrêmes minimale et maximale $; \mathrm{F}$ de SNEDECoR significatif au seuil $0,05^{*}, 0,01^{* *}, 0,001^{* * *}$

Test de KRAMER = les valeurs affectées par la même lettre ne diffèrent pas statistiquement entre elles au seuil 0,05 .

$\mathrm{m}=$ mean $;(\mathrm{p})=$ maximum and minimum values $; \mathrm{F}$ significant at $\mathrm{P}=0.05^{*}, 0.01^{*}, 0.001^{* * *}$.

Values with the same letter are not significantly different at $\mathrm{P}=0.05$ according to Kramer's test.

TABLEAU 4

Etude de la formation des femelles d'Heterodera avenae sur des haploïdes doublés issus du blé Athys. Production of females of Heterodera avenae on doubled haploids issued from wheat cv. Athys.

\begin{tabular}{|c|c|c|c|c|c|c|}
\hline \multirow[b]{2}{*}{ Plante testée } & \multicolumn{3}{|c|}{$24 \mathrm{Fr} 2$} & \multicolumn{3}{|c|}{$48 \mathrm{Ha} 2$} \\
\hline & Nombre de grains testés & Femelles & s/Plante & Nombre de grains testés & Femelles & s/plantes \\
\hline ATHYS & 9 & 3,33 & $(1-6)$ & 9 & 5,44 & $(1-8)$ \\
\hline HD (ATHYS) $1 \mathrm{~g} 3$ & 11 & 4,45 & $(1-9)$ & 11 & 6,64 & $(1-10)$ \\
\hline HD (ATHYS) 2 g2 & 10 & 5,00 & $(2-9)$ & 8 & 5,62 & $(2-10)$ \\
\hline
\end{tabular}

$m=$ moyenne $;(p)=$ valeurs extrêmes minimale et maximale.

$\mathrm{m}=$ mean $;(\mathrm{p})=$ minimum and maximum values.

2. Etude de matériel variable pour son caractère hôte $v i s$-à-vis d'Heterodera avenae

Lors d'une première année d'essai, des plantes de la lignée de blé V.P.M. 1.1.1.2. R4 sont testées en boîtes de Petri vis-à-vis du pathotype Ha41. Parmi elles, 3 ont été retenues en raison de leurs niveaux de multiplication différents du nématode soit 0,4 et 5 femelles 
pour respectivement les numéros : 12,49 et 37 . Immédiatement après ce test, les plantes sont repiquées sur sol pour assurer leur épiaison et reproduction.

Au cours de l'année de culture suivante, entre 50 et 60 grains pris au hasard parmi les descendants de chacun des 3 numéros sont testés vis-à-vis du même pathotype Ha41 (tabl. 5). La formation moyenne de femelles par plante de chaque lignée va en croissant suivant l'ordre obtenu l'année précédente à savoir successivement les numéros 12,49 et 37 . Et le numéro 37 produit significativement plus de femelles que les 2 autres. La lignée V.P.M. 1.1.1.2 R4 ne semble donc pas fixée pour son caractère hôte vis-à-vis d' $H$. avenae.

TABLEAU 5

Etude de la formation des femelles d'Heterodera avenae, pathotype Ha41, sur blé V.P.M. 1.1.1.2 R4 non fixé pour son caractère hôte vis-à-vis du nématode.

Production of females of Heterodera avenae (pathotype Ha4l) on the wheat V.P.M. 1.1.1.2 R4 variable for host quality to the nematode.

\begin{tabular}{cccc}
\hline $\begin{array}{c}\text { Origine du végétal } \\
\text { étudié }\end{array}$ & $\begin{array}{c}\text { Nombre de grains } \\
\text { testés }\end{array}$ & \multicolumn{2}{c}{$\begin{array}{c}\text { Femelles formées/ } \\
\text { plantes }\end{array}$} \\
\hline V.P.M. $\mathrm{n}^{\circ} 12$ & 58 & $6,24^{\mathrm{b}}$ & $(\mathrm{p})$ \\
V.P.M. $\mathrm{n}^{\circ} 49$ & 50 & $6,94^{\mathrm{b}}$ & $(2-14)$ \\
V.P.M. $\mathrm{n}^{\circ} 37$ & 58 & $8,12^{\mathrm{a}}$ & $(1-14)$ \\
& analyse de variance & $\mathrm{F}_{165}^{2}=6,005^{* *}$ \\
\hline
\end{tabular}

$\mathrm{m}=$ moyenne $;(\mathrm{p})=$ valeurs extrêmes minimale et maximale ; $F$ de SNEDECOR significatif au seuil $0,01^{* *}$.

Test de Kramer = les valeurs affectées par la même lettre ne diffèrent pas statistiquement entre elles au seuil 0,05 .

$\mathrm{m}=$ mean $;(\mathrm{p})=$ maximum and minimum values $; \mathrm{F}$ significant at $\mathrm{P}=0.01^{* *}$.

Values with the same letter are not significantly different at $P=0.05$ according to Kramer's test.

\section{Influence du nématode}

Le génotype du nématode peut être modifié, soit par pression de sélection, soit par recombinaison suite à la réalisation de croisements entre pathotypes. Ces 2 facteurs seront analysés successivement.

\section{Influence de la pression de sélection (tabl. 6, fig. 3)}

L'élevage de nématodes appartenant au pathotype Fr2 a été fait 2 fois consécutives sur l'un des 2 cultivars suivant, le blé « Hardi », ou l'orge "Ortolan » présentant des gènes de résistance à l'encontre d'autres pathotypes d' $H$. avenae. Le nombre de femelles formées au cours de la $2^{\mathrm{e}}$ culture donne par ordre décroissant les combinaisons : "Hardi »/ " Hardi » supérieure à " Hardi »/ «Ortolan » supérieure à " Ortolan »/« Hardi » ou « Ortolan »/« Ortolan ».

2. Influence de la recombinaison: utilisation d'hybrides interpathotypes (tabl. 7, fig. 4).

Des hybrides $\mathrm{F} 1$ de première génération, issus du croisement entre femelles Ha41 et mâles Hal 2 sont élevés sur le blé Lutin avec, soit $48 \mathrm{~L} 2$, soit $24 \mathrm{~L} 2$ par boîtes de Petri et des températures de stockage de 14-
TABLEAU 6

Etude de pression de sélection effectuée à l'aide $d u$ pathotype $\mathrm{Fr} 2 d$ 'Heterodera avenae.

Study of selection pressure applied with pathotype Fr2 of Heterodera avenae.

\begin{tabular}{cccc}
\hline $\begin{array}{c}\text { Origine } \\
\text { du nématode }\end{array}$ & $\begin{array}{c}\text { Nombre de grains } \\
\text { testés }\end{array}$ & \multicolumn{2}{c}{$\begin{array}{c}\text { Femelles } \\
\text { formées/plantes }\end{array}$} \\
\hline $\begin{array}{c}\text { Multiplication sur } \\
\text { Ortolan }\end{array}$ & & $\mathrm{m}$ & $(\mathrm{p})$ \\
ORTOLAN & 33 & $3,45^{\mathrm{c}}$ & $(1-10)$ \\
HARDI & 39 & $4,62^{\mathrm{b}}$ & $(1-9)$ \\
Multiplication sur & & & \\
Hardi & & & \\
HARDI & 40 & $5,85^{\mathrm{a}}$ & $(3-11)$ \\
ORTOLAN & 39 & $3,46^{\mathrm{c}}$ & $(1-8)$ \\
& analyse de variance & $\mathrm{F}_{147}^{3}=11,425^{* * *}$ \\
\hline
\end{tabular}

$\mathrm{m}=$ moyenne $;(\mathrm{p})=$ valeurs extrêmes minimale ou maximale $;$ $F$ de SNEDECOR significatif au seuil $0,001^{* * *}$.

Test de Kramer = les valeurs affectées par la même lettre ne diffèrent pas statistiquement entre elles au seuil 0,05 .

$\mathrm{m}=$ mean $;(\mathrm{p})=$ maximum and minimum values $; \mathrm{F}$ significant at $\mathrm{P}=0.001^{* * *}$

Values with the same letter are not significantly different at $\mathbf{P}=0.05$ according to Kramer's test.
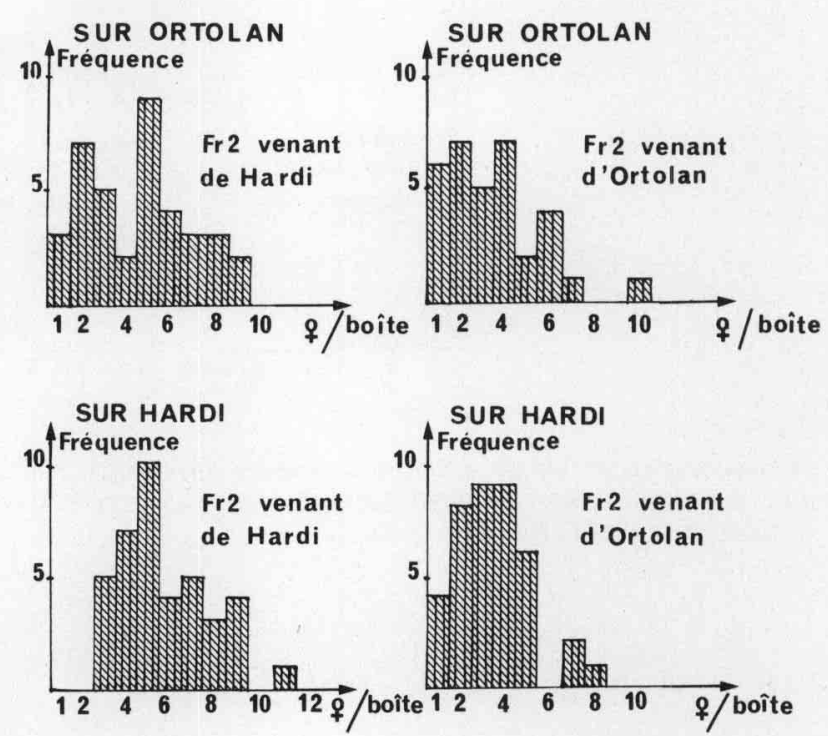

Figure 3

Fréquence d'apparition de nombres de femelles formées par plante après pression de sélection effectuée avec le pathotype Fr 2 d'Heterodera avenae.

Frequency of appearance of numbers of females developed per plant after selection pressure with pathotype Fr2 of Heterodera avenae.

$16{ }^{\circ} \mathrm{C}$ ou $14-19^{\circ} \mathrm{C}$. Mis dans les conditions de température optimale de développement de chacun de leurs parents et à quantités d'inoculum égales correspondant à celles utilisées actuellement dans nos tests, les hybrides F1 forment significativement plus de femelles que leurs parents. Nous notons cependant avec 24 larves infectieuses par plante que la formation des femelles hybrides $\mathrm{F} 1$ s'effectue mieux à $14-19{ }^{\circ} \mathrm{C}$, températures favorisant le développement du pathotype méridional Ha41 qu'à $14-16{ }^{\circ} \mathrm{C}$. Elevés à $14-16^{\circ} \mathrm{C}$ ces hybrides F1 fournissent statistiquement autant de femelles que des nématodes Ha41 placés à $14-19{ }^{\circ} \mathrm{C}$. 
TABLEAU 7

Etude de l'agressivité d'hybrides issus de croisements entre pathotypes Ha4l et Hal2 d'Heterodera avenae sur le blé Lutin démuni de tout gène de résistance vis-à-vis du nématode.

Study of the aggressiveness of hybrids issued from crosses between pathotypes Ha4l and Hal2 of Heterodera avenae on wheat $c v$. Lutin without resistance genes against the nematode.

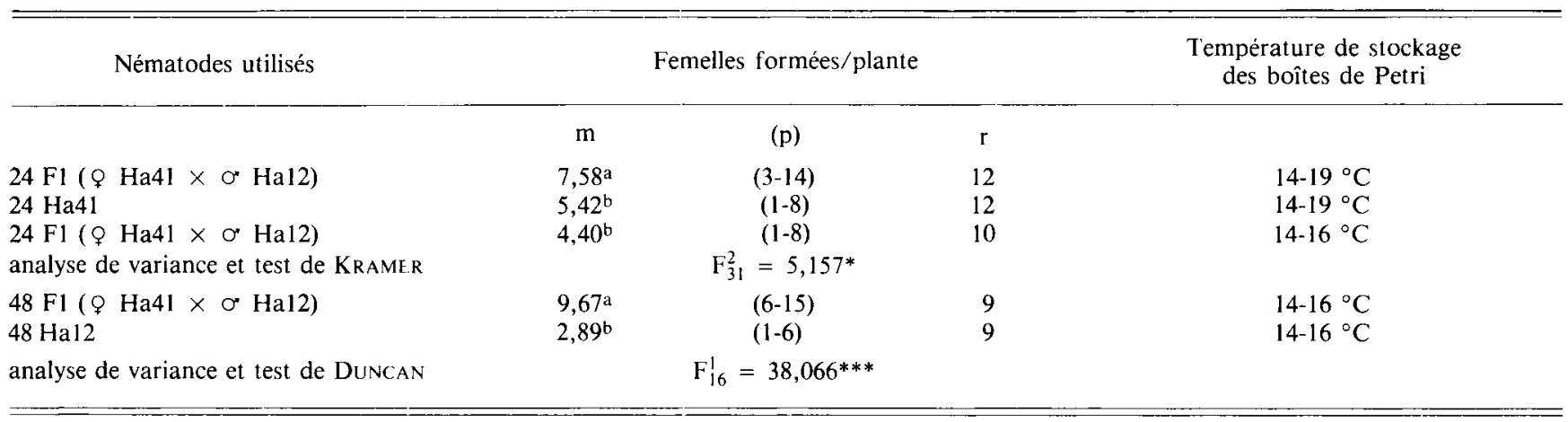

$\mathrm{m}=$ moyenne, $(\mathrm{p})=$ valeurs extrêmes minimale et maximale $; \mathrm{F}$ de SNEDECOR significatif au seuil $0,05^{*}$ et $0,001^{* * *}$.

Tests de KRAmER et de DUNCAN = les valeurs affectées par des lettres différentes ne sont pas statistiquement identiques au seuil 0,05 . $\mathrm{m}=$ mean $;(\mathrm{p})=$ maximum and minimum values $; \mathrm{F}$ significant at $\mathrm{P}=0.05^{*}$ and $0.001^{* * *}$.

Values with the same letter are not significantly different at $P=0.05$ according to Kramer's test and Duncan's test.

Figure 4

Fréquence d'apparition de nombres de femelles formées par plante après hybridation entre pathotypes d'Heterodera avenae et mise en élevage sur le blé $c v$. Lutin.

\section{DISCUSSION - CONCLUSION}

\section{A. Les facteurs n'agissant pas sur la formation des femelles}

\section{Facteurs liés à la méthode}

Pour l'ensemble des pathotypes et des cultivars de blé et d'orge considérés, le facteur position de la boîte de Petri dans la pile n'a jamais été responsable de formations de femelles en nombres statistiquement différents. La quantité de lumière sans doute peu différente pour les 4 niveaux de plantes A, B, C et D (fig. 1) n'influe pas sur le nombre de femelles formées (tabl. 1). Les variations notées entre les maxima thermiques des 4 types de boîte pour l'élevage de chacun des 3 pathotypes n'ont pas d'effet sur l'importance des populations femelles développées. Les températures d'élevage du nématode correspondent à des conditions excellentes de développement des 3 pathotypes (PERSON \& DOUSSINAULT, 1978). Cependant, pour compa-
Frequency of appearance of numbers of females developed per plant after crosses between pathotypes of Heterodera avenae and multiplication on wheat $c v$. Lutin. rer la formation des femelles d'un pathotype sur diverses variétés, il est plus prudent d'effectuer l'étude sur des plantes de chaque cultivar réparties équitablement entre les 4 positions possibles A, B, C et D. Par ce moyen, nous éviterons tout biais de l'expérimentation pouvant être introduit par un choix systématique d'un type de boîte pour une variété donnée et différent de ceux utilisés pour les autres cultivars testés.

\section{Facteurs liés au végétal}

L'emploi simultané de plusieurs lots de grains issus de différentes plantes génotypiquement identiques et provenant d'un même haploïde doublé tel que HD (Moisson) 2 est à l'origine d'un nombre moyen de femelles similaire, comme on pouvait s'y attendre (tabl. 3). Leur utilisation démontre seulement que les écarts importants enregistrés entre les nombres de femelles formées sur chaque répétition d'un même lot de grains ne proviennent pas du patrimoine génétique du végétal et ne peuvent être attribués qu'aux facteurs nématode ou méthode. 
Des lots de grains provenant de différents haploïdes tels que HD (Moisson) 1 et HD (Moisson) 2 pour Ha41 et HD (Athys) 1 et HD (Athys) 2 pour Fr2 et Ha12, produisent des nombres de femelles de chaque pathotype statistiquement identiques (tabl. 3 et 4). Les résultats enregistrés sur ces haploïdes sont également non différents de ceux obtenus sur leurs lignées d'origine respectives. Les haploïdes utilisés ont donc le même génotype que leur lignée d'origine pour leur capacité d'hôte vis-à-vis de chacun des pathotypes d'H. avenae.

\section{B. Les facteurs agissant sur la formation des femelles}

\section{Les facteurs liés à la méthode}

Le poids du grain n'est intervenu qu'une seule fois, dans le cadre de l'étude du cultivar «Capitole» et du pathotype Ha41 (tabl. 2). Assez curieusement le nombre de femelles est corrélé négativement au poids du grain. Cette observation reste difficile à expliquer.

La différence significative enregistrée entre populations femelles Ha41 obtenues sur des plantes génotypiquement identiques de l'haploïde doublé HD (César) 1 étudiées en $6^{\mathrm{e}}$ ou $7^{\mathrm{e}}$ génération d'autofécondation correspond sans doute à des conditions de cultures différentes lors de l'obtention de ces 2 générations (tabl. 3). Aucune corrélation n'a été notée entre nombre de femelles obtenues et poids du grain, mais globalement la formation des femelles paraît affectée par les mauvaises conditions de culture des plantes de la $6^{\mathrm{e}}$ génération qui se traduisent sur la qualité de la semence.

\section{Les facteurs liés au végétal}

L'utilisation de matériel végétal non fixé pour le caractère plante hôte, tel que V.P.M. 1.1.1.2 R4 pouvant posséder des gènes de résistance issus de l'Ae. ventricosa lignée 11 vis-à-vis d' $H$. avenae entraîne une variabilité dans la formation des femelles du nématode. Dans ce cas, la résistance doit être polygénique et chaque plante peut posséder ou non un ou plusieurs des gènes de résistance vis-à-vis du nématode.

\section{Les facteurs liés au nématode}

Les hybrides issus de croisements entre femelles Ha41 et mâles Ha 2 forment plus de femelles que leurs parents sur blé dépourvu de gènes de résistance à l'égard d'H. avenae (tabl. 7) et sont donc, dans ce cas, plus agressifs que les pathotypes d'origine. Il pourrait $\mathrm{y}$ avoir un effet hétérosis chez les individus $\mathrm{F} 1$ qui se traduirait par une plus grande vigueur du nématode et une augmentation de son agressivité. Par ailleurs, la virulence ou capacité de ces hybrides à se multiplier sur des cultivars d'orge tels que «Siri », «Ortolan», «Vogue » et «P31.322.1 », présentant des gènes de résistance vis-à-vis d' $H$. avenae, ne s'exprime pas du fait de la présence chez eux de gènes de virulence récessifs à l'état hétérozygote (PERSON \& RIVOAL, 1979). L'absence de virulence vis-à-vis de certaines variétés semble donc être compensée par un gain en agressivité sur d'autres cultivars. Un tel type d'observation a déjà été fait sur les pathotypes français d' $H$. avenae. Ainsi Ha41 se multipliant peu ou pas sur variétés d'avoine est plus agressif vis-à-vis des cultivars de blé et d'orge démunis de tout gène de résistance connus à l'égard d' $H$. avenae que Ha12 particulièrement prolifique sur avoine (RIVOAL \& PERSON-DEDRYVER, 1982).

Le développement de Fr2 durant un seul cycle sur l'orge «Ortolan » possédant des gènes de résistance vis-à-vis de $\mathrm{Ha} 41$ et Fr3 uniquement, rend le nématode moins agressif vis-à-vis d'autres plantes possédant ou non des gènes de résistance à son égard (tabl. 6). A partir de cet exemple nous pouvons avancer l'hypothèse suivante en ce qui concerne $H$. avenae : l'utilisation effective de la capacité d'un pathotype à contourner des résistances se traduit par une diminution globale de son agressivité.

Tous ces faits ne sont pas surprenants, ils se retrouvent chez d'autres agents pathogènes. Ainsi les recombinaisons chez Venturia inaequalis, agent de la tavelure du pommier, sont à l'origine de l'apparition de pathotypes plus agressifs que les souches sauvages (AMORIM, 1984). MAKounZI \& Chevaugeon (1979) obtiennent des résultats identiques avec la pyriculariose du riz (Pyricularia oryzae Briosi et Cav.). Il est également démontré que les individus de $P$. oryzae qui possèdent le plus de capacités à contourner les résistances sont aussi ceux qui sont les moins agressifs vis-à-vis du riz (CHEVAugeon et al., 1978).

\section{Conclusions}

Notre travail prouve que l'utilisation de la technique "Boîtes de Petri » est particulièrement bien adaptée à l'étude de l'agressivité d' $H$. avenae. Les seuls facteurs méthodologiques susceptibles d'intervenir significativement sur la formation des femelles concernent parfois le poids des grains utilisés et donc très probablement les conditions de culture des plantes produisant ces grains. Il est cependant facile de réduire l'influence de ces facteurs, en choisissant préférentiellement pour chaque expérience une variété donnée des grains de même calibre et provenant de la culture la mieux réalisée.

Du point de vue matériel végétal, seules des lignées non fixées présentant une résistance polygénique à l'encontre du parasite peuvent être à l'origine de modifications importantes de la formation des femelles.

En conséquence, les facteurs devant influer sur les variations globales de l'importance des populations femelles d' $H$. avenae dépendent essentiellement du nématode lui-même et de ses potentialités d'agressivité. L'existence de pathotypes, possédant une agressivité propre vis-à-vis d'une variété donnée, a déjà été démontrée (RIVOAL \& PERSON-DEDRYVER, 1982). Nous avons constaté au cours de cette étude que cette fluctuation de l'agressivité peut encore augmenter par recombinaisons génétiques après croisements entre pathotypes, ou par pression de sélection sur plantes possédant des gènes de résistance à l'égard du nématode. Il semblerait même que le nématode puisse perdre en agressivité ce qu'il a gagné en virulence. Cette observation est particulièrement intéressante, car nous pouvons supposer qu'en cas de contournement de la résistance par $H$. avenae, les individus plus virulents que les pathotypes déjà répertoriés se multiplieraient, de toutes façons moins qu'eux, sur variétés démunies de gènes de résistance. 
Quand on a pris le soin de réduire l'effet des facteurs méthodologiques, les variations de l'agressivité notée entre chaque répétition d'un même essai, sont donc imputables au génotype du nématode lui-même. Les défauts de pénétration ou de développement des larves infectieuses L2 par suite de mortalité pourraient également être incriminés mais la part prise par ces 2 fac- teurs reste limitée. Le taux de pénétration des larves L2 se maintient à 80 p. 100 d'une plante à une autre d'un même essai (MUGNIERY \& PERSON, 1976). Il en est de même du taux de mortalité de ces larves qui est très faible dans tous les cas.

Reçu le 9 mars 1987 Accepté le 23 juillet 1987.

\section{RÉFÉRENCES BIBLIOGRAPHIQUES}

Amorim L., 1984. Contribution à l'analyse des épidémies de la tavelure du pommier. Stabilité des résistances génétiques de l'hôte et valeurs adaptatives des pathotypes. Thèse de Docteur-Ingénieur, Univ. Paris-Sud, Centre d'Orsay, 109 p.

Andersen S., Andersen K., 1982. Suggestions for determination and terminology of pathotypes and genes for resistance in cyst-forming nematodes, especially Heterodera avenae. EPPO Bull., 12, 379-386.

Chevaugeon J., Bah C. P., Makounzi J. A., 1978. Mesure rapide de la force des gènes de résistance. Application à la Pyriculariose du riz. Ann. Phytopathol., 10, 277-286.

Dosba F., Doussinault G., 1978. Création de lignées de blé présentant les caractéristiques agronomiques favorables d'Aegilops ventricosa. Ann. Amélior. Plant., 28, 31-35.

Dosba F., Rivoal R., 1981. Les lignées d'addition Blé et Aegilops ventricosa Tausch. II. Etude de leur comportement et de celui de leurs progéniteurs vis-à-vis d'Heterodera avenae Woll. Agronomie, 1, 559-564.

Dosba F., Doussinault G., Person F., Rivoal R., 1979. Sélection de céréales résistantes à Heterodera avenae : cas du blé. $17^{e} \mathrm{col}$. Soc. Française Phytopathol., 7 décembre 1979. Paris. Poster.

Kramer C. Y., 1956. Extension of multiple range tests to group means with unequal numbers of replications. Biometrics, 12, 307310.

Makounzi J. A., Chevaugeon J., 1979. Recombinaison mitotique et variations de l'agressivité chez Pyricularia oryzae Briosi el Cav. Ann. Phytopathol., 11, 145-156.

Mugniery D., Person F., 1976. Méthode d'élevage de quelques nématodes à kyste du genre Heterodera. Sci. agronom., Rennes, 1976, 217-220.

Parisi L., Picard E., 1986. Disease response of doubled haploid lines and their original cultivars in wheat (Triticum aestivum L.). $Z$. Pflanzenzüchtg., 96, 63-78.
Person F., Doussinault G., 1978. Influence de la température et des caractères des races d'Heterodera avenae Woll. sur la validité d'un test en conditions contrôlées, utilisable en sélection des céréales. Ann. Amélior. Plant., 28, 513-527.

Person F., Rivoal R., 1979. Hybridation entre les races Frl et Fr4 d'Heterodera avenae Woll. en France et étude du comportement d'agressivité des descendants F1. Revue Nématol., 2, 177-183.

Person-Dedryver F., Doussinault G., 1984. Interactions génétiques entre pathotypes français d'Heterodera avenae Woll. et variétés d'orge. I. - Aspect variétal. Agronomie, 4, 763-771.

Rivoal R., 1977. Identification des races biologiques du nématode à kystes des céréales, Heterodera avenae Woll. en France. Ann. Zool. Ecol. Anim., 9, 261-272.

Rivoal R., 1979. Biologie d'Heterodera avenae Wollenweber en France. II. Etude des différences dans les conditions thermiques d'éclosion des races Fr1 et Fr4. Rev. Nématol., 2, 233-248.

Rivoal R., Person-Dedryver F., 1982. Caractérisation des pathotypes d'Heterodera avenae en France : influence de la période de cul ture sur le pouvoir discriminant de cultivars d'Avena sativa et différences dans la capacité à former des femelles. EPPO Bull., 12, 387 391.

Rivoal R., Person F., Caubel G., Scotto La Massese C., 1978. Méthodes d'évaluation de la résistance des céréales au développement des nématodes: Ditylenchus dipsaci, Heterodera avenae et Pratylenchus spp. Ann. Amélior. Plant., 28, 31-54.

Rode A., Hartmann C., Benslimane A., Picard E., Quetier F., 1987. Gametoclonal variation detected in the nuclear ribosomal DNA from doubled haploid lines of a spring wheat (Triticum aestivum L., cv. "César »). Theor. Appl. Genet., 74, 31-37. 